Apidologie, 1987, 18 (4), 305-320

\title{
INFLUENCE DE Z'AGE DES ABEILLES (APIS MELLIFICA L.) ET DE LA CHALEUR SUR LE COMPORTEMENT DE VARROA JACOBSONI OUD.
}

\author{
Yves LE CONTE et Gérard ARNOLD \\ Laboratoire de Neurobiologie Comparée des Invertébrés \\ I.N.R.A-C.N.R.S., 91440 Bures-sur-Yvette
}

\section{RÉSUMÉ}

L'attractivité différentielle des abeilles pour Varroa jacobsoni en fonction de l'âge et de la température, a été étudiée d'une part au moyen d'un test qui met en évidence le transfert des varroas depuis des abeilles parasitées vers des abeilles indemnes et d'autre part au moyen d'un test d'olfactométrie adapté à l'étude comparée des effets de la chaleur et des odeurs.

Les résultats ont montré que :

1) les varroas quittent rapidement les abeilles naissantes pour des abeilles âgées de plus de 2 jours ;

2) la chaleur ambiante et la chaleur produite par l'abeille ont un rôle déterminant dans la répartition des varroas sur des abeilles d'âges différents ;

3) des stimulus chimiques et/ou vibratoires interviennent également dans la répartition du varroa sur les abeilles ;

4) à la température ambiante de $23^{\circ} \mathrm{C}$, une augmentation de chaleur de $1,2^{\circ} \mathrm{C}$ est un stimulus plus efficace que des abeilles vivantes ou que les autres stimulus testés (chimiques, vibratoires).

\section{INTRODUCTION}

Varroa jacobsoni Oud., Acarien ectoparasite d'Apis mellifica, vivait initialement en parasite équilibré sur Apis cerana, une abeille du continent asiatique (Delfinado, 1963). Chez cette espèce, la survie des colonies n'est pas en péril car les varroas femelles ne se reproduisent pas dans le couvain d'ouvrières (KoENIGER et al., 1981). L'introduction d'Apis mellifica dans les pays asiatiques, ainsi que le commerce des essaims et des reines, ont favorisé l'infestation de cette espèce par Varroa jacobsoni. Les colonies d'Apis mellifica sont détruites après 3 ou 4 ans d'infestation (RITTER, 1981).

La majorité des pays européens est aujourd'hui touchée par la parasitose, et depuis son apparition en France en 1982 (Colin et al., 1983), 60 départements sont officiellement reconnus infestés à la date d'août 1987 (La Santé de l'Abeille, 1987). 
Les résultats présentés ici s'intègrent dans un programme de recherche qui vise à comprendre les mécanismes impliqués dans les relations abeille-varroa, afin de mettre au point une lutte intégrée et ainsi de limiter l'emploi des acaricides.

Les signaux émis par l'abeille, et qui pourraient favoriser l'attraction du varroa, sont de plusieurs types : chimiques, thermiques, vibratoires, etc. Les résultats établis précédemment ont montré une attraction du parasite par des abeilles à une distance de 0,5 à $1 \mathrm{~cm}$ (Le ConTe et al., 1984). Par ailleurs, le fait que la quantité de chaleur dégagée par l'abeille varie en fonction de l'âge (RoTH, 1965) nous a conduit à proposer l'hypothèse que la production de chaleur par l'abeille pourrait constituer l'un des facteurs déterminants de l'attraction de Varroa jacobsoni par celle-ci.

Les données présentées ci-dessous concernent l'étude de l'attractivité différentielle de Varroa jacobsoni en fonction de l'âge des abeilles, ainsi que le rôle déterminant des facteurs thermiques dans cette répartition.

\section{MATÉRIELS ET MÉTHODES}

L'attraction de Varroa jacobsoni a été analysée d'une part au moyen d'un test de choix en cagette d'élevage permettant d'observer la répartition des varroas dans des lots d'abeilles d'âges différents, et, d'autre part, au moyen d'un test d'olfactométric à diffusion passive des odeurs.

\section{A. Expériences de choix}

40 abeilles parasitées et 40 abeilles non parasitées, toutes d'âge connu, sont marquées (extrémité d'une aile sectionnée) et placées dans une cagette $(6 \mathrm{~cm} \times 5 \mathrm{~cm} \times 4 \mathrm{~cm})$, soit dans un volume de $120 \mathrm{~cm}^{3}$, ce qui correspond environ à la densité de population dans la ruche. Après un temps fixé en fonction du type d'expérience, les varroas sont dénombrés sur chacune des 80 abeilles afin de connaître la répartition des parasites. Les expériences sont réalisées en lumière artificielle, à une température de $25 \pm 0,5^{\circ} \mathrm{C}$, et une humidité relative de $70 \pm 5 \%$.

Dans de telles conditions, plusieurs facteurs sont étudiés :

1. Influence de l'âge des abeilles sur la répartition des varroas

1) Transfert spontané des varroas sur des abeilles d'une même classe d'âge

L'expérience est réalisée avec des abeilles parasitées et des abeilles non parasitées de même âge. Des abeilles naissantes, et des abeilles de $1,2,4,6,8,10,12$ et 14 jours sont successivement testées. Des essais préliminaires ont conduit à fixer la durée des tests à douze heures.

2) Transfert spontané des varroas vers des abeilles d'âges différents

Deux séries de tests sont réalisées :

1) mise en présence d'abeilles naissantes parasitées avec des abeilles de $0,1,2,4,6,8,10,12$ ou 14 jours, non parasitées ;

2) mise en présence d'abeilles naissantes non parasitées avec des abeilles de $0,1,2,4,6,8,10,12$ ou 14 jours, parasitées.

La durée de l'expérience est fixée à 12 heures. 


\section{Vitesse de transfert des varroas}

L'expérience est réalisée avec des abeilles naissantes parasitées et des abeilles de 5 jours non parasitées, en faisant varier la durée de mise en présence des deux groupes d'abeilles.

\section{Influence de la température ambiante}

Le transfert des varroas des abeilles naissantes vers des abeilles de 5 jours est analysé en faisant varier la température ambiante. Des tests préliminaires ont conduit à fixer la durée de l'expérience à une heure et à douze heures. La température ambiante varie de 21 à $34,5^{\circ} \mathrm{C}$ (soit $21,25,28,31$ et $\left.34,5 \pm 0,5^{\circ} \mathrm{C}\right)$.

\section{B. Expérience d'olfactométrie}

Le dispositif est constitué d'une boîte de Pétri en verre de $90 \mathrm{~mm}$ de diamètre, dont le fond est percé de 4 trous de $12 \mathrm{~mm}$ de diamètre, placés chacun aux quatre points cardinaux et à $2,2 \mathrm{~cm}$ du centre de la boîte.

Dans chaque trou s'emboîte un tube de verre de $3 \mathrm{~cm}$ de longueur, recouvert à ses 2 extrémités par de la gaze. Un bouchon de grillage de $0,5 \mathrm{~cm}$ d'épaisseur est inséré sous la gaze supérieure pour éviter tout contact entre les varroas et les abeilles. Les abeilles à tester sont placées à l'intérieur de deux des tubes, les deux autres tubes sont utilisés soit vides comme témoins, soit avec des abeilles d'âge différent. Ce dispositif a été adapté à l'étude des effets de la chaleur sur l'attraction du varroa. Pendant l'expérience, des thermocouples de type YSI 401 (précision $\pm 0,1^{\circ} \mathrm{C}$ ) sont placés à l'intérieur de chaque tube. La température ambiante ainsi que la température des différents tubes sont relevées en début d'expérience puis toutes les 5 minutes.

Dix varroas sont placés au centre de la boîte de Pétri. Le nombre de varroas situés sur la gaze de chaque tube est relevé $1,5,10,15,20$ minutes après leur introduction. Le pourcentage moyen des varroas présents pour chaque catégorie de tube est calculé à partir des 5 relevés. L'expérience est répétée au minimum 4 fois, en tournant la boîte de 45 degrés à chaque fois, afin de limiter l'effet des facteurs d'environnement. Un pourcentage global est établi à partir des pourcentages moyens (4, au minimum). Le dispositif est nettoyé entre chaque répétition, d'abord avec un détergent (Teepol), puis à l'alcool à $70^{\circ}$, et rincé ensuite à l'eau distillée. Les expériences sont réalisées à la lumière naturelle et dans une humidité relative de $60 \% \pm 5 \%$.

Des tests d'attraction et des tests de choix sont réalisés afin de déterminer la part qu'il convient d'attribuer respectivement à l'âge de l'abeille et à la température.

\section{1) Test d'attraction}

Deux tubes contiennent chacun deux abeilles de même âge. Les deux autres, utilisés comme témoins, sont vides.

Des abeilles de deux classes d'âge, abeilles naissantes et abeilles de 5 jours, sont testées séparément, à une température ambiante de $24^{\circ} \mathrm{C}$.

\section{2) Test de choix}

Dans une première série d'expériences, 2 tubes contiennent deux abeilles de 5 jours et les 2 autres tubes contiennent soit 2 abeilles naissantes, soit une source de chaleur.

Dans une deuxième série d'expériences, la gaze recouvrant l'extrémité supérieure des 4 tubes est remplacée par un morceau de papier d'aluminium qui empêche la diffusion des stimulus chimiques volatils émis par l'abeille. Cinq expériences sont réalisées avec des abeilles de 2 classes d'âge différentes : abeilles naissantes-abeilles de 1 jour, émergentes-5 jours, émergentes- 11 jours, 1 jour- 11 jours, 11 jours30 jours. 
Dans une troisième série d'expériences, le dispositif expérimental étant identique au précédent (deuxième expérience), deux des 4 tubes sont chauffés à l'aide d'une résistance électrique placée dans chacun des tubes, les deux autres tubes contiennent chacun deux abcilles de 30 jours.

La température ambiante est de $23 \pm 0,5^{\circ} \mathrm{C}$ pour chaque séric.

Après avoir établi le pourcentage global des varroas placés sur chaque catégorie de tubes, les données obtenues ont été traitées par le test statistique de comparaison de pourcentages (Test $t$ de Student, tables de l'écart réduit), ou par le test du $\chi^{2}$ selon le cas.

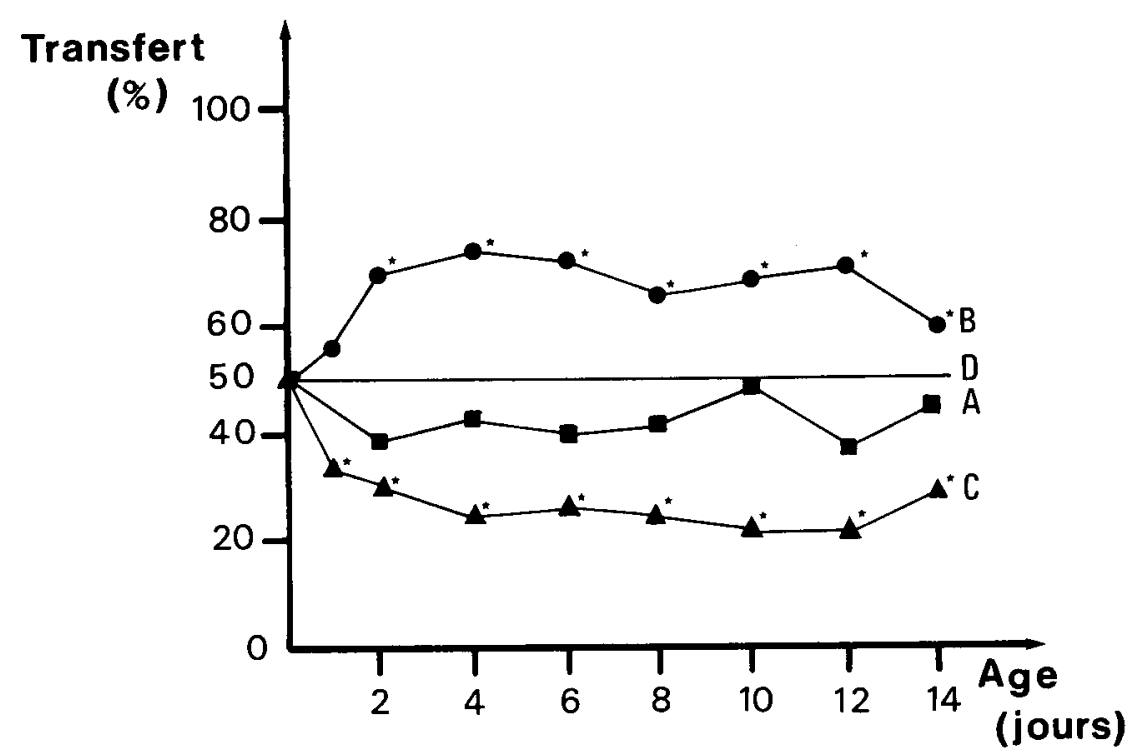

Fig. 1. - Pourcentage de transfert des varroas des abeilles parasitées vers des abeilles non parasitées en fonction de l'âge des abeilles

A : Pourcentage de transfert depuis les ouvrières parasitées vers les ouvrières non parasitées de même âge.

B : Pourcentage de transfert depuis les ouvrières émergentes vers les ouvrières plus âgées.

C : Pourcentage de transfert depuis les ouvrières âgées vers les ouvrières émergentes.

$\mathrm{D}$ : Taux de répartition homogène théorique $(50 \%)$.

* Différence significative par rapport à une distribution homogène.

FIG. 1. - Rate of varroa passage from parasitized bees to unparasitized ones in relation to bee age

A : Rate of passage from parasitized bees toward unparasitized bees and bees of the same age.

B : Rate of passage from newly emerged bees toward older ones.

C : Rate of passage from older bees toward newly emerged bees.

D : Rate of equal movement.

* Significant difference in relation to an equal distribution.

$X$ - axis : Age of bees (days).

$Y$ - axis : Rate of passage (\%). 


\section{RÉSULTATS}

A. Expérience de choix

I. Influence de l'âge des abeilles sur la répartition des varroas

1) Transfert spontané des varroas sur des abeilles d'une même classe d'âge

La répartition des varroas dans les deux classes d'abeilles de même âge n'est pas significativement différente (test du $\chi^{2}$ ) d'une répartition homogène (50\% des varroas dans chaque classe) (fig. 1).

2) Transfert spontané des varroas sur des abeilles d'âges différents

Les résultats sont représentés sur la figure 1.

En présence d'abeilles d'au moins 2 jours, les varroas quittent les abeilles naissantes pour parasiter les abeilles plus âgées $(P<0,001)$.

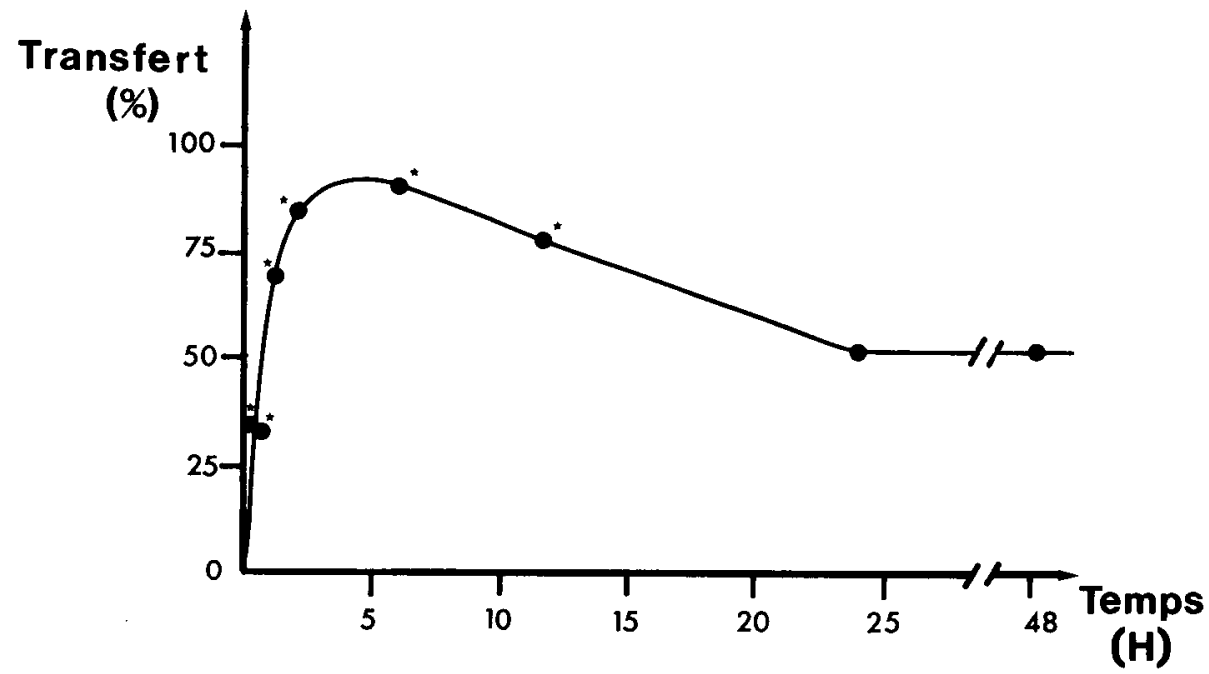

FIg. 2. - Rôle de la durée de l'expérience sur le pourcentage de transfert des varroas des abeilles naissantes vers des abeilles de 5 jours

* Différence significative par rapport à une distribution homogène.

FIG. 2. - Rate of varroa passage from newly emerged bees to 5-day-old bees in relation to duration of experiment.

$X$ - axis : Duration of experiment (hours).

$Y$ - axis : Rate of passage (\%).

* Significant difference in relation to an equal distribution. 
Un faible pourcentage de varroas (environ $20 \%$ ) passe des abeilles âgées vers les abeilles naissantes; la majorité des varroas restent donc sur les abeilles les plus âgées $(\mathrm{P}<0,001)$. La répartition des parasites sur les abeilles diffère significativement lorsque les abeilles les plus âgées ont au moins 1 jour.

En outre, si on place des abeilles naissantes parasitées dans une cagette, entre 50 et $55 \%$ des varroas quittent les abeilles et se déplacent sur les parois de la cagette après une heure. Certains parasites arrivent même à sortir de la cagette, et ce, quelque soit la température ambiante (de 18 à $34,5^{\circ} \mathrm{C}$ ). $\mathrm{Ce}$ phénomène ne se produit pas lorsque l'expérience est réalisée avec des abeilles parasitées âgées de plus de 2 jours.

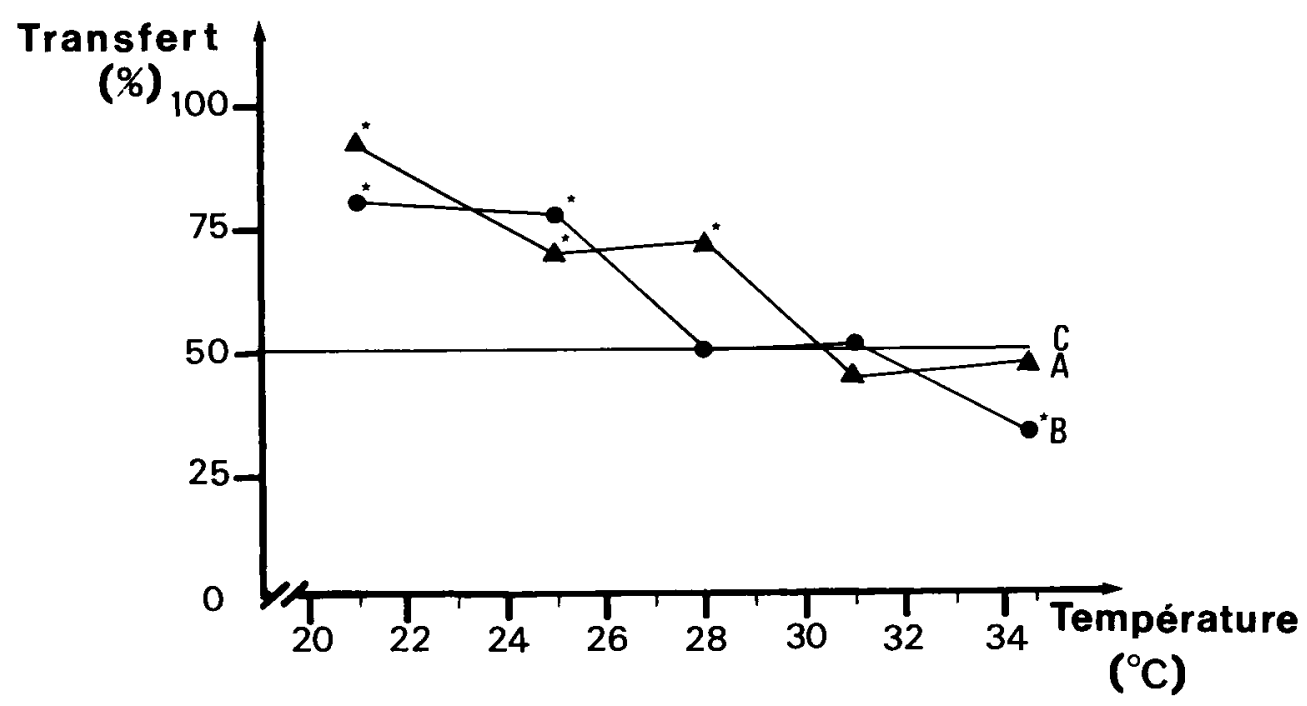

FIG. 3. - Rôle de la température ambiante sur le pourcentage de transfert des varroas à partir d'abeilles naissantes vers des abeilles de 5 jours

A : 1 heure d'expérience.

B : 12 heures d'expérience.

$C$ : ligne de répartition homogène.

* Différence significative par rapport à une distribution homogène.

FIG. 3. - Rate of passage from newly emerged bees to 5-day-old bees in relation to ambient temperature

A : 1 hour experiment.

B : 12 hour experiment.

$C$ : Line of equal movement.

$\mathrm{X}$ - axis : Ambient temperature $\left({ }^{\circ} \mathrm{C}\right)$.

$Y$ - axis : Rate of passage (\%).

* Significant difference in relation to an equal distribution. 


\section{Vitesse de transfert des varroas}

Les varroas passent rapidement des abeilles naissantes vers les abeilles de 5 jours (Fig. 2) ; ainsi après une heure d'expérience, $70 \%$ des varroas se trouvent sur les abeilles de 5 jours. Si l'expérience est prolongée pendant 24 heures ou 48 heures, on obtient alors une répartition homogène des varroas, mais les 2 lots d'abeilles ont alors atteint respectivement l'âge de 1 ou 2 jours et 6 ou 7 jours.

\section{Influence de la température ambiante}

Le pourcentage de transfert des varroas à partir des abeilles naissantes vers les abeilles de 5 jours diminue lorsque la température ambiante augmente (Fig. 3). Une répartition homogène des varroas entre les deux catégories d'abeilles d'âges différents est obtenue à partir d'une température ambiante de $30^{\circ} \mathrm{C}$ environ, pour une durée d'expérience supérieure à une heure.

\section{B. Tests d'olfactométrie}

Au cours de ces expériences, les varroas qui sont déposés au centre de la boîte de Pétri, ont un comportement de recherche permanent et s'arrêtent fréquemment pendant quelques secondes. S'ils sont attirés par un tube et son contenu, ils se déplacent alors sur la gaze et s'immobilisent.

La quantité de chaleur produite par une abeille est calculée en divisant l'augmentation de chaleur dans le tube par le nombre d'abeille (2).

\section{1) Test d'attraction}

Les varroas sont attirés par les abeilles à une distance minimale de 0,5 à $1 \mathrm{~cm}$. L'attraction par les abeilles de 5 jours est significativement plus importante (Test $t, P<0,001$ ) que celle exercée par les abeilles naissantes (Tabl. 1).

La chaleur produite par les abeilles de 5 jours (en moyenne 1,5 degré par rapport au témoin) est plus importante que celle produite par les abeilles naissantes (en moyenne 0,7 degré).

En outre, il apparaît que $80 \%$ des varroas environ ont fait leur choix après 5 minutes d'expérience.

\section{2) Test de choix}

Les abeilles de 5 jours attirent significativement plus les varroas que les abeilles naissantes. On observe une différence de production de chaleur de $1,3{ }^{\circ} \mathrm{C}$ entre les deux catégories d'abeilles (Tabl. 2). 
ТАВ. 1. - Test d'attraction de varroa par des abeilles naissantes ou des abeilles de 5 jours (deux abeilles par tube)

Température ambiante $=24^{\circ} \mathrm{C}$ (les chiffres entre parenthèses représentent

la différence de température à l'intérieur de chaque tube par rapport à la température ambiante)

TABL. 1. - Attraction of varroa by newly emerged bees and by 5-day-old bees (two bees per tube) Ambient temperature $=24^{\circ} \mathrm{C}$ (numbers in brackets give the difference of heat inside each tube in comparison to ambient temperature)

\begin{tabular}{l|c|c|c|c}
\hline $\begin{array}{c}\text { Age des abeilles } \\
\text { Age of bees }\end{array}$ & $\begin{array}{c}\text { Nombre } \\
\text { de varroas } \\
\text { testés } \\
\text { Number of varroa }\end{array}$ & $\begin{array}{c}\text { Nombre moyen } \\
\text { de varroas attirés } \\
\text { par les abeilles } \\
(\%) \\
\text { Mean number } \\
\text { of varroa attracted } \\
\text { by bees }(\%)\end{array}$ & $\begin{array}{c}\text { Nombre moyen } \\
\text { de varroas sur } \\
\text { les tubes vides } \\
\text { (témoins) } \%) \\
\text { Mean number } \\
\text { of varroa in empty } \\
\text { tubes }(\text { controls) } \\
(\%)\end{array}$ & $\begin{array}{c}\text { Signification } \\
\text { statistique } \\
\text { Statistical } \\
\text { significance }\end{array}$
\end{tabular}

TAB. 2. - Test d'attraction comparée de varroa entre abeilles de 5 jours et abeilles naissantes (deux abeilles par tube)

TABL. 2. - Comparison of attractiveness between older and newly emerged bees (two bees per tube)

\begin{tabular}{c|c|c|c}
\hline \hline $\begin{array}{c}\text { Nombre de varroas } \\
\text { testés } \\
\begin{array}{c}\text { Number of varroa } \\
\text { tested }\end{array}\end{array}$ & $\begin{array}{c}\text { Nombre moyen } \\
\text { de varroas attirés } \\
\text { par les abeilles } \\
\text { de 5 jours }(\%) \\
\text { Mean number } \\
\text { of varroa } \\
\text { attracted by 5-day-old } \\
\text { bees }(\%)\end{array}$ & $\begin{array}{c}\text { Nombre moyen } \\
\text { de varroas attirés } \\
\text { par les abeilles } \\
\text { naissantes }(\%) \\
\text { Mean number } \\
\text { of varroa } \\
\text { attracted by newly } \\
\text { emerged bees }(\%)\end{array}$ & $\begin{array}{c}\text { Signification statistique } \\
\text { Statistical significance }\end{array}$ \\
\hline 70 & $\begin{array}{c}59,7 \\
\left(+1,9^{\circ} \mathrm{C}\right)\end{array}$ & $\begin{array}{c}3,9 \\
\left(+0,6^{\circ} \mathrm{C}\right)\end{array}$ & $\mathrm{P}<0,001$ \\
\hline
\end{tabular}

Si les tubes sont bouchés, ce qui bloque la diffusion des stimulus chimiques émis par les abeilles sans empêcher la diffusion de la chaleur, l'attraction des varroas vers les tubes contenant les abeilles d'âges différents est significativement plus grande lorsque la différence de chaleur à l'intérieur des tubes est en moyenne supérieure ou égale à $1,8^{\circ} \mathrm{C}$ par rapport à la température ambiante (Tabl. 3).

De même les tubes chauffés sont plus attractifs que les tubes fermés contenant les abeilles (Tabl. 4) et une différence de température d'au moins $+1,1^{\circ} \mathrm{C}$ attire les varroas préférentiellement aux différents stimulis émis par les abeilles dans ces conditions expérimentales. 
ТАB. 3. - Test de choix entre des abeilles d'âges différents dans des tubes bouchés (deux abeilles par tube)

TABL. 3. - Comparison of attractiveness between bees of different ages in closed tubes (two bees per tube)

\begin{tabular}{|c|c|c|c|c|}
\hline $\begin{array}{l}\text { Age des abeilles } \\
\text { Age of bees }\end{array}$ & $\begin{array}{c}\text { Nombre } \\
\text { de varroas } \\
\text { testés } \\
\text { Number of varroa }\end{array}$ & $\begin{array}{c}\text { Nombre moyen } \\
\text { de varroas attirés } \\
\text { par les abeilles } \\
\text { plus jeunes (\%) } \\
\text { Mean number } \\
\text { of varroa attracted } \\
\text { by younger bees } \\
(\%)\end{array}$ & $\begin{array}{c}\text { Nombre moyen } \\
\text { de varroas attirés } \\
\text { par les abeilles } \\
\text { plus âgées (\%) } \\
\text { Mean number } \\
\text { of varroa attracted } \\
\text { by older bees } \\
(\%)\end{array}$ & $\begin{array}{l}\text { Signification } \\
\text { statistique } \\
\text { Statistical } \\
\text { significance }\end{array}$ \\
\hline $\begin{array}{l}\text { Naissantes } / 1 \text { jour } \\
\text { Newly emerged } / 1 d\end{array}$ & 40 & $\begin{aligned} & 19 \\
&\left(+0,6^{\circ} \mathrm{C}\right) \\
&\end{aligned}$ & $\begin{array}{r}38,5 \\
\left(+1,7^{\circ} \mathrm{C}\right) \\
\end{array}$ & N.S. \\
\hline $\begin{array}{l}\text { Naissantes } / 5 \text { jours } \\
\text { Newly emerged } / 5 d\end{array}$ & 40 & $\begin{array}{l}17 \\
\left(+0,6^{\circ} \mathrm{C}\right) \\
\end{array}$ & $\begin{array}{c}58 \\
\left(+1,8^{\circ} \mathrm{C}\right)\end{array}$ & $\mathrm{P}<0,001$ \\
\hline $\begin{array}{l}\text { Naissantes } / 11 \text { jours } \\
\text { Newly emerged/11 } d\end{array}$ & 40 & $\begin{array}{l}15 \\
\left(+0,5^{\circ} \mathrm{C}\right) \\
\end{array}$ & $\left(\begin{array}{l}62 \\
\left(+1,8^{\circ} \mathrm{C}\right)\end{array}\right.$ & $\mathrm{P}<1,001$ \\
\hline $\begin{array}{l}1 \text { jour } / 11 \text { jours } \\
1 \mathrm{~d} / 11 \mathrm{~d}\end{array}$ & 40 & $\begin{array}{l}29 \\
\left(+0,8^{\circ} \mathrm{C}\right)\end{array}$ & $\begin{array}{l}47 \\
\left(+1,1^{\circ} \mathrm{C}\right)\end{array}$ & N.S. \\
\hline $\begin{array}{l}11 \text { jours } / 30 \text { jours } \\
11 \mathrm{~d} / 30 \mathrm{~d}\end{array}$ & 40 & $\begin{array}{l}22 \\
\left(+1,0^{\circ} \mathrm{C}\right) \\
\end{array}$ & $\left(\begin{array}{l}71 \\
\left(+2,2^{\circ} \mathrm{C}\right)\end{array}\right.$ & $P<0,001$ \\
\hline $\begin{array}{l}\text { Témoin/Témoin } \\
\text { control/control }\end{array}$ & 40 & $\begin{array}{c}4,8 \\
\left(0^{\circ} \mathrm{C}\right)\end{array}$ & $\begin{array}{c}5,5 \\
\left(0^{\circ} \mathrm{C}\right)\end{array}$ & N.S. \\
\hline
\end{tabular}

TAB. 4. - Test de choix entre abeilles de 30 jours et une source de chaleur placées dans des tubes bouchés (deux abeilles par tube)

TABL. 4. - Comparison of attractiveness between 30-day-old bees and heat in closed tubes ( $t$ wo bees per tube)

\begin{tabular}{|c|c|c|c|c|}
\hline $\begin{array}{c}\text { Nombre } \\
\text { de varroas } \\
\text { testés } \\
\text { Number of varroa } \\
\text { tested }\end{array}$ & $\begin{array}{c}\text { Nombre moyen } \\
\text { de varroas attirés } \\
\text { par les abeilles } \\
(\%) \\
\text { Mean number } \\
\text { of varroa attracted } \\
\text { by bees }(\%)\end{array}$ & $\begin{array}{l}\text { Nombre moyen } \\
\text { de varroas attirés } \\
\text { par les tubes } \\
\text { chauffés (\%) } \\
\text { Mean number } \\
\text { of varroa attracted } \\
\text { by warmed } \\
\text { tubes (\%) }\end{array}$ & $\begin{array}{c}\text { Différence } \\
\text { de température } \\
\text { entre les tubes } \\
\left({ }^{\circ} \mathrm{C}\right) \\
\text { Temperature } \\
\text { difference between } \\
\text { tubes }\left({ }^{\circ} \mathrm{C}\right)\end{array}$ & $\begin{array}{l}\text { Signification } \\
\text { statistique } \\
\text { Statistical } \\
\text { significance }\end{array}$ \\
\hline 40 & $\begin{array}{c}10,5 \\
\left(+1,7^{\circ} \mathrm{C}\right)\end{array}$ & $\begin{array}{c}42,5 \\
\left(+8,5^{\circ} \mathrm{C}\right)\end{array}$ & 6,8 & $P<0,01$ \\
\hline 80 & $\begin{array}{c}9,5 \\
\left(+2,1^{\circ} \mathrm{C}\right)\end{array}$ & $\begin{array}{c}62,5 \\
\left(+7,4^{\circ} \mathrm{C}\right)\end{array}$ & 5,3 & $\mathrm{P}<0,001$ \\
\hline 40 & $\begin{array}{c}5,5 \\
\left(+1,9^{\circ} \mathrm{C}\right)\end{array}$ & $\begin{array}{c}63 \\
\left(+6,1^{\circ} \mathrm{C}\right)\end{array}$ & 4,2 & $P<0,001$ \\
\hline 40 & $\begin{array}{c}10,5 \\
\left(+1,9^{\circ} \mathrm{C}\right)\end{array}$ & $\begin{array}{c}48,5 \\
\left(+3,0^{\circ} \mathrm{C}\right)\end{array}$ & 1,1 & $P<0,001$ \\
\hline
\end{tabular}


TAB. 5. - Test de choix entre des abeilles de 30 jours et une source de chaleur placées dans des tubes ouverts (deux abeilles par tube)

TABL. 5. - Comparison of attractiveness between 30-day-old bees and heat in open tubes (two bees per tube)

\begin{tabular}{c|c|c|c|c}
\hline \hline $\begin{array}{c}\text { Nombre } \\
\text { de varroas } \\
\text { testés }\end{array}$ & $\begin{array}{c}\text { Nombre moyen } \\
\text { de varroas attirés } \\
\text { par les tubes } \\
\text { chauffés }(\%) \\
\text { Mean number } \\
\text { of varroa attracted } \\
\text { by warmed } \\
\text { tubes }(\%)\end{array}$ & $\begin{array}{c}\text { Nombre moyen } \\
\text { de varroas attirés } \\
\text { par les abeilles } \\
(\%) \\
\text { Mean number } \\
\text { of varroa attracted } \\
\text { by bees }(\%)\end{array}$ & $\begin{array}{c}\text { Différence } \\
\text { de température } \\
\text { entre les tubes } \\
\left({ }^{\circ} \mathrm{C}\right) \\
\text { Temperature } \\
\text { difference between } \\
\text { tubes }\left({ }^{\circ} \mathrm{C}\right)\end{array}$ & $\begin{array}{c}\text { Signification } \\
\text { statistique } \\
\text { Statistical } \\
\text { significance }\end{array}$ \\
\hline 310 & $\begin{array}{c}60,6 \\
\left(+7^{\circ} \mathrm{C}\right)\end{array}$ & $\begin{array}{c}2,5 \\
\left(+1,4^{\circ} \mathrm{C}\right)\end{array}$ & 5,6 & $\mathrm{P}<0,001$ \\
\hline 83 & $\begin{array}{c}34,5 \\
\left(+2^{\circ} \mathrm{C}\right)\end{array}$ & 1,2 & $\mathrm{P}<0,001$ \\
\hline 40 & $\begin{array}{c}51,3 \\
\left(+1,5{ }^{\circ} \mathrm{C}\right)\end{array}$ & 1,5 & $\mathrm{P}<0,5$ \\
\hline \hline
\end{tabular}

A la température ambiante de $23^{\circ} \mathrm{C}$, une différence de température de $+1,2^{\circ} \mathrm{C}$ en moyenne, créée par une source de chaleur, est suffisante pour attirer les varroas préférentiellement aux tubes ouverts contenant les abeilles (Tabl. 5).

\section{DISCUSSION-CONCLUSION}

Selon les stades, les varroas se trouvent soit sur les abeilles adultes, soit sur le couvain (larves et nymphes). Les femelles varroas pénètrent dans les cellules contenant des larves d'abeilles quelques heures avant leur operculation (IFANTIDIs, 1983). Le couvain de mâles est infesté environ 8 fois plus que celui d'ouvrières (Schulz, 1984 ; Sulimanovic et al., 1982).

Chez les abeilles adultes, il semble que les acariens infestent à la fois les ouvrières et les mâles (SCHNEIDER, 1985). Toutefois étant donné le nombre très supérieur d'ouvrières par rapport aux mâles dans une colonie, les varroas se répartissent évidemment beaucoup plus sur les ouvrières.

Chez celles-ci, la répartition des varroas n'est pas homogène mais dépend de l'âge. Les résultats présentés ici confirment les résultats préliminaires qui avaient montré que les varroas étaient peu attirés par les abeilles naissantes, mais préféraient des abeilles âgées d'au moins deux jours (LE ConTE et al., 1984). 
Chez des ouvrières encagées de plus de deux jours, la répartition des varroas ne semble pas dépendre de l'âge des abeilles. Par contre, dans la ruche, les acariens semblent préférer les nourrices aux butineuses dans plus de $60 \%$ des cas (Schneider, 1985 ; Kraus et al., 1986).

$\mathrm{Au}$ laboratoire, les études olfactométriques ont montré que les varroas étaient attirés à une distance comprise entre 0,5 et $1 \mathrm{~cm}$ aussi bien par les insectes (ouvrière, mâle, reine) que par la cire provenant du nid à couvain ou des opercules (Le Conte et ARnold, 1986).

La reconnaissance de l'hôte (abeille), et qui plus est de son stade (larve, adulte) et de son sexe (ouvrière, mâle), implique l'utilisation par le varroa d'au moins trois types de signaux, des signaux chimiques, thermiques et/ou vibratoires.

Les facteurs chimiques interviennent puisque non seulement la cire du nid à couvain et la cire d'opercules des cellules qui contiennent le couvain sont attractives, mais également des ouvrières et des mâles morts (LE CONTE et al., 1984). Les facteurs attractifs contenus dans la cire peuvent d'ailleurs provenir des abeilles elles-mêmes. En outre, des ouvrières nourrices mortes demeurent plus attractives que des butineuses mortes (Kraus et al., 1986).

Des signaux vibratoires interviennent également, mais ne sont pas prédominants dans l'attraction des varroas par l'abeille (Le Conte et ARNold, 1986).

Les facteurs chimiques et vibratoires ne semblent cependant pas être seuls en cause dans l'attraction des varroas. Ainsi, si les varroas ont le choix entre un compartiment contenant une abeille (âgée de 30 jours) et un compartiment contenant uniquement une source de chaleur supérieure d'au moins $1,2^{\circ} \mathrm{C}$ à la chaleur produite par l'abeille, environ la moitié des varroas se dirigent dans le compartiment le plus chaud et $10 \%$ dans celui contenant l'ouvrière. Ce choix se produit aussi bien lorsque l'abeille est séparée du varroa par de la gaze, qui laisse passer les signaux chimiques, thermiques et vibratoires émis par l'abeille, que lorsque l'ouvrière est séparée du varroa par un écran de papier d'aluminium qui ne laisse passer que les signaux thermiques et vibratoires.

Les facteurs thermiques sont donc susceptibles de jouer un grand rôle lors de l'attraction du varroa par l'abeille. Ainsi, lorsqu'on parasite des abeilles naissantes avec des varroas et qu'on introduit ensuite des abeilles plus âgées, les varroas quittent en moins d'une heure les abeilles les plus jeunes tant que la température ambiante est inférieure à $28^{\circ} \mathrm{C}$. Par contre quand la température est supérieure à $28^{\circ} \mathrm{C}$, la répartition devient homogène entre les abeilles d'âges différents. Le passage des varroas sur les abeilles plus âgées serait dû, au moins en partie, à des facteurs thermiques. 
Ce point semble confirmé par le fait que les abeilles naissantes produisent moins de chaleur que les abeilles plus âgées. L'augmentation de la production de chaleur en fonction de l'âge de l'abeille est conforme aux données de Roth (1965). Dans nos expériences, à la température ambiante de $23^{\circ} \mathrm{C}$, une abeille naissante fait monter la température de $0,28^{\circ} \mathrm{C}$ en moyenne dans son enceinte, alors que pour une abeille âgée de 11 jours, l'augmentation est de $0,65^{\circ} \mathrm{C}$. De même, lorsqu'on donne aux varroas le choix entre des abeilles de 11 jours et des abeilles de 30 jours, qui produisent une augmentation de température de $1,1^{\circ} \mathrm{C}$ par abeille, les varroas se dirigent vers les abeilles les plus âgées.

Les résultats apparaissent en contradiction avec ceux obtenus avec des abeilles vivant en colonies où les nourrices (en général âgées de 3 à 12 jours) sont plus attractives que les butineuses (en général âgées d'au moins 21 jours) (KRAus et al., 1986). Mais la contradiction n'est peut être qu'apparente car ces auteurs n'ont pas mesuré la quantité de chaleur dégagée comparativement par les nourrices et les butineuses.

Le thermopréférendum du varroa, qui est compris entre $31,3{ }^{\circ} \mathrm{C}$ et $34,2^{\circ} \mathrm{C}$ selon la température ambiante (Le ConTE et ARNOLD, 1986), correspond bien à la température du couvain $\left(34^{\circ} \mathrm{C}\right.$ pour le couvain d'ouvrière et $31^{\circ} \mathrm{C}$ pour le couvain de mâle) et à la température du corps des ouvrières $\left(32,4^{\circ} \mathrm{C}\right.$ sur le thorax et $31^{\circ} \mathrm{C}$ sur l'abdomen) (HeinRich, 1980). Les résultats contenus dans ce travail montrent que les varroas sont capables de détecter une différence de température de $1,1^{\circ} \mathrm{C}$.

Chez l'acarien Ornithodoros concanensis, la température $\left(40^{\circ} \mathrm{C}\right)$ semble être le principal stimulus qui déclenche l'attraction vers l'hôte (oiseau, chauvesouris) à une distance de 1 à $2 \mathrm{~cm}$, alors que la géotaxie négative et la perception sonore de l'hôte semblent impliquées dans la recherche de l'hôte à plus longue distance (jusqu'à 12 à $15 \mathrm{~m}$ ). Chez les Ixodoidae, la chaleur et l'odeur seraient également les principaux stimulus responsables de la perception de l'hôte par ces acariens (BALASKov, 1972 ; WebB, 1979).

Chez certains acariens, des sensilles thermoréceptrices sont présentes sur les tarses des pattes antérieures (WaLlaDE et al., 1981).

Chez le varroa, des recherches anatomiques et électrophysiologiques sont actuellement en cours pour mettre en évidence les thermorécepteurs.

Le fait que, dans une population d'abeilles naissantes, $50 \%$ des varroas quittent les abeilles quelle que soit la température ambiante semble indiquer soit une absence d'attraction, soit une répulsion des varroas pour ces jeunes abeilles. Des stimulus autres que la chaleur pourraient donc être impliqués.

Il convient maintenant de déterminer avec précision la nature et l'importance relative des autres types de signaux émis par les abeilles, en fonction du 
sexe, du stade et de l'âge, qui interviendraient dans l'attraction de varroa. Parmi ceux-ci, les stimulus chimiques et les stimulus vibratoires jouent probablement un rôle important (Le ConTe et ARnold, 1986).

Reçu pour publication en mai 1987. Accepté pour publication en août 1987.

\title{
REMERCIEMENTS
}

Les auteurs remercient MM. Y. Bouchery, P. Robert et M. Stengel de la Station de Zoologie de l'I.N.R.A. de Colmar, ainsi que M. R. CAnTEneur pour leur accueil et leur aide au cours de ce travail.

\author{
SUMMARY \\ THE EFFECTS OF BEE AGE AND OF HEAT \\ ON THE PARASITIC BEHAVIOR OF VARROA JACOBSONI
}

The difference in attractiveness of bees of different ages to Varroa mites and the significant role of heat on the movement of mites among bees were studied through two different behavioral tests:

- in the first test the passage of mites from a group of 40 bees to another group of 40 unparasitized bees was observed (Tabl. 1-5, Fig. 1-3). Bee ages, time of experiment and room temperature were varied during the test;

- the second experiment was conducted using an olfactometer with passive diffusion of odor. It was made of a Petri dish pierced with 4 holes at the bottom. A glass tube closed at the bottom by a piece of gauze and at the top by a piece of gauze and a piece of aluminum foil, was encased in each hole. According to the experiment, some tubes contained bees, some were warmed and others were empty (controls). Mites were placed in the center of the Petri dish. The number of mites found on each category of tubes was counted in relation to time. Thermic sounding lines were used to check tube temperature.

Results showed that :

1) Varroa mites leave newly emerged bees rapidly in order to go to 2-day-old bees.

2) Room temperature and temperature produced by bees have a determinant role on mite movement among bees of different ages.

3) At room temperature $\left(23^{\circ} \mathrm{C}\right)$, an increase of $1.2{ }^{\circ} \mathrm{C}$ in tubes is more attractive to Varroa than living bees and more attractive than other stimuli (chemical, vibratory, thermic, etc.) produced by bees.

4) Chemical or/and vibratory stimuli seem to be involved in the movement of mites among bees.

\section{ZUSAMMENFASSUNG}

\author{
EFFEKT VOM ALTER DER BIENEN UND VON WÄRME \\ AUF DAS PARASITIERUNGSVERHALTEN DER VARROA-MILBE
}

Durch zwei verschiedene Verhaltenstests wurde der Unterschied in der Attraktivität von Bienen verschiedenen Alters auf Varroa-Milben sowie der Einfluß von Wärme auf die Verteilung der Milben auf den Bienen untersucht : 
Im ersten Test wurde das Überwechseln der Milbe von einer Gruppe von 40 Bienen auf eine andere Gruppe von 40 unparasitierten Bienen beobachtet (Tab. 1-5, Abb. 1-3). Während der Versuche wurden Alter der Bienen, Versuchsdauer und Umgebungstemperatur verändert.

Beim zweiten Test wurde ein Olfaktometer mit passiver Verteilung des Geruches benutzt. Es bestand aus einer Petrischale, deren Boden mit vier Löchern versehen war. In jedes der Löcher wurde ein Glasröhrchen eingesetzt, das an seiner unteren Öffnung mit einem Stück Gaze, an seiner oberen Öffnung mit einem Gazestück und einer Aluminiumfolie verschlossen war. Je nach dem Versuch, enthielten die Röhrchen Bienen, oder sie wurden gewärmt oder sie blieben als Kontrolle leer und unbehandelt. In die Mitte der Petrischale wurden die Varroa-Milben gesetzt. Die Zahl der Milben in jeder Kategorie von Röhrchen, abhängig von der Zeit, wurde gezählt. Die Temperatur in den Röhrchen wurde mit Temperaturfühlern gemessen.

Die Resultate zeigen folgendes :

1) Dic Varroa-Milben wechseln rasch von frisch geschlüpften zu zwei Tage älteren Bienen über.

2) Die Umgebungstemperatur und die von Bienen erzeugte Wärme haben einen entscheidenden Einfluß auf die Verteilung der Milben auf Bienen verschiedenen Alters.

3) Bei einer Umgebungstemperatur von $23{ }^{\circ} \mathrm{C}$ ist einc Erhöhung von $1{ }^{\circ} \mathrm{C}$ an den Röhrchen für die Varroas stärker attraktiv als lebende Bienen oder andere Reize (chemisch, Vibration, thermisch usw.), die von Bienen erzeugt werden.

4) Chemische oder/und Vibrationsreize scheinen bei der Verteilung der Varroa-Milben auf Bienen beteiligt zu sein.

\section{BIBLIOGRAPHIE}

Balaskov Y.S., 1972. - Bloodsucking ticks (Ixodoidac) - Vectors of discases of man and animals. Misc. Publ. Entomol. Soc. Am., 8, 160-376.

Colin M.E., Faucon J.P., Heinrich A., Ferry R. et Giauffret A., 1983. - Elude du premier foyer français de varroatose de l'abeille. Bull. Acad. Vét. de France, 56, 89-93.

Delfinado M., 1963. - Mites of the honeybee in South-East Asia. J. Apic. Res., 2, 113-114.

HeinRich B., 1980. - Mecanisms of body-temperature regulation in honeybees, Apis mellifera. II. Regulation of thoracic temperature at high air temperatures. J. Exp. Biol., 85, 73-87.

Heinrich B., 1981. - The mecanisms and energetics of honeybee swarm temperature regulation. J. Exp. Biol., 91, 25-55.

IfANTIDIS M.D., 1983. - Ontogenesis of the mite Varroa jacobsoni in worker and drone honeybec brood cells. J. Apic. Res., 22 (3), 200-206.

Koeniger N., Koeniger G., Wijayagunasekara N.H.P., 1981. - Beobachtungen über die Anpassung von Varroa jacobsoni an ihren natürlichen Wirt Apis cerana in Sri Lanka. Apidologie, 12 (1), 37-40.

Kraus B., Koeniger N. und Fuchs S., 1986. - Unterscheidung zwischen Bienen verschiedenen Alters durch Varroa jacobsoni Oud. und Bevorzugung von Ammenbienen im Sommerbienenvolk. Apidologie, 17 (3), 257-266.

La Santé de l'Abeille, 1987. - Epidémiologie de la Varroase en France, (100), 193.

le Conte Y., Arnold G., Chauffalle J., Masson C., 1984. - Role of semiochemicals in HoncybeeVarroa relationships : preliminary data. 2nd Meeting of the E.C. varroa Experts'group, Thessaloniki (Grèce), 26-28 sept. 1984 (communication orale).

Le Conte Y., Arnold G., 1986. - Implied sensory signals in the Honeybee-Varroa relationships. 3nd Meeting of the E.C. varroa Experts'group, Bad Homburg (R.F.A.), (communication orale) (sous presse).

RIrter W., 1981. - Varroa disease of the honeybee Apis mellifera. Bee World, 62 (4), 141-153.

Roтн M., 1965. - La production de chaleur chez Apis mellifera L. Ann. Abeille, 8 (I), 5-77. 
Schnejder P., 1985. - Befall von Sammlerinnen, Stockbienen, Flugdrohnen und Stockdrohnen mit Varroa jacobsoni. Apidologie, 16 (3), 209-211.

Schulz A., 1984. - Reproduktion und Populationsentwicklung der parasitischen Milbe Varroa jacobsoni Oud. in Abhängigkeit vom Brutzyklus ihre Wirtes Apis mellifera L. Apidologie, 15 (4), 401-420.

Sulimanovic D., Ruttner F., Pechiacker H., 1982. - Studies on the biology of reproduction in Varroa jacobsoni. Honeybee Sci., 3 (3), 109-112.

Waladde S.M., Kokwaro E.D. and Chimtawi M., 1981. - A cold receptor on the tick, Rhipicephalus appendiculatus : electrophysiological and ultrastructural observations. Insect Sci. Applications, 1, 191196.

WeBB J.P., 1979. - Host-locating behavior of nymphal Ornithodoros concanensis (Acarina : Argasidae). J. Med. Entomol., 16, 5, 437-447. 